\title{
- Protective levels of canine distemper virus antibody in an urban dog population using plaque reduction neutralization test
}

\author{
O.I. OYEDELE ${ }^{1 *}$, D.O. OLUWAYELU², S.I.B. CADMUS ${ }^{2}$, S.O. ODEMUYIWA ${ }^{1}$ and F.D. ADU ${ }^{1}$
}

\begin{abstract}
OYEDELE, O.I., OLUWAYELU, D.O., CADMUS, S.I.B., ODEMUYIWA, S.O. \& ADU, F.D. 2004. Protective levels of canine distemper virus antibody in an urban dog population using plaque reduction neutralization test. Onderstepoort Journal of Veterinary Research, 71:227-230

Blood samples from 50 dogs were collected at three veterinary clinics in Ibadan and Abuja, Nigeria and the serum from each sample was evaluated serologically for neutralizing antibodies against canine distemper virus (CDV) by the highly sensitive plaque reduction (PRN) neutralization assay. Thirteen dogs had plaque reduction neutralization titres of $0-100$, seven had titres of 100-1000 while 30 had titres ranging from 1000-6 000. The PRN titres of vaccinated dogs were found to be significantly higher than unvaccinated dogs. The widespread use of the highly reproducible PRN test for the evaluation of antibody response to CDV may be very important in the generation of international CDV positive serum standards that should help to improve pre-and post-vaccination testing of dogs worldwide.
\end{abstract}

Keywords: Antibodies, canine distemper, dogs, plaque reduction neutralization

\section{INTRODUCTION}

Canine distemper, caused by canine distemper virus (CDV) of the genus Morbillivirus, is the most important viral disease of dogs that is characterized by high morbidity and mortality in unvaccinated canine populations worldwide. It is a highly infectious, acute or subacute febrile disease of dogs and other carnivores which has been known since 1760 (Fenner, Gibbs, Murphy, Rott, Studdert \& White 1993). The advent of preventive vaccination programmes has gone a long way to reduce the incidence of the

Author to whom correspondence is to be directed. Present address: c/o Dr O.O. Oyedele, School of Anatomical Sciences, Faculty of Health Sciences, University of the Witwatersrand, Johannesburg, 2001 South Africa

1 Department of Virology, University College Hospital, Ibadan, Nigeria

2 Faculty of Veterinary Medicine, University of Ibadan, Ibadan, Nigeria

Accepted for publication 8 July 2003-Editor disease in recent years (McCaw, Thompson, Tate, Bonderer \& Chen 1998).

According to Horst (1975), the most effective method of controlling canine distemper is mass vaccination of dogs and other carnivores. Most commercial canine distemper vaccines are made from the Onderstepoort strain of the virus isolated in South Africa (Yoshida, Shin, Iwatsuki, Gemma, Miyashita, Tomonaga, Hirayama, Mikami \& Kai 1999). However, strains different from the Onderstepoort strain have also been isolated (Iwatsuki, Miyashita, Yoshida, Gemma, Shim \& Mori 1999; Wakasa, Iwatsuki, Ohashi, Nakamura \& Kai 2000). In Nigeria, commercially available CDV vaccines are marketed and administered in combination with hepatitis, leptospirosis, parvovirus and parainfluenza vaccines (DHLP+P combined vaccine).

It is recommended that puppies be given a series of vaccinations to stimulate active immunity as maternally derived immunity declines. This should then 
be followed by annual revaccination to maintain immunity (Greene 1990). However, the usefulness of annual revaccination of vaccinated dogs is still widely debated. Smith (1995) has suggested that a more cost-effective and beneficial approach is to first measure serum antibody titres to determine if revaccination is necessary.

The availability and widespread use of attenuated virus vaccines appears to have controlled the disease in most countries. In a routine survey of veterinary clinics in Nigeria the prevalence of canine distemper was reported to have become infrequent (Oyedele, unpublished data 2001). Although previous studies have positively correlated CDV-neutralizing antibody levels with immunity in dogs (Greene 1990), the reproducibility and predictive values of serum neutralization test and its usefulness in the quantitation of antibody response have been very controversial (Soma, Ishii, Hara, Yamamoto, Yoshida, Kinoshita \& Nomura 2001). Conversely, several studies have shown that the plaque reduction neutralization (PRN) test is more sensitive, specific and reproducible for the quantitation of antibody responses to many viral infections, including canine distemper (Shope 1990; Lee, Cohen, Hand \& Nokes 1999; Soma et al. 2001). This study was therefore designed to determine the prevalence of neutralizing antibodies to CDV in dogs in the population in order to evaluate the CDV vaccination programme in Nigeria and determine risk of infection using the highly sensitive and reproducible PRN test.

\section{MATERIALS AND METHODS}

\section{Sample collection}

Blood samples were collected from 50 dogs at three veterinary clinics, namely the State veterinary clinic in Ibadan and two private veterinary clinics in Ibadan and Abuja which are major urban centres in Nigeria. The dogs were bled by standard venipuncture and serum obtained and stored frozen at $-20^{\circ} \mathrm{C}$ until analyzed.

The records of vaccination, if any, were obtained from the owners of the dogs and verified from clinic records. All dogs with unverifiable vaccination records were considered as unvaccinated and were so grouped.

\section{Canine distemper virus stock}

Canine distemper virus (Onderstepoort strain) was obtained from the laboratory of Dr C.P. Muller of the
Immunology division, Laboratoire National de Sante, Luxembourg. The virus was seeded at low multiplicity of infection (MOI 0.001) on a confluent Vero cell monolayer. Culture supernatant collected at peak cytopathic effect was centrifuged at $1200 \mathrm{~g}$ for $5 \mathrm{~min}$ at $4{ }^{\circ} \mathrm{C}$ and filtered through a $0.2 \mu \mathrm{m}$ syringe filter (Millipore, Bedford, Massachusetts, USA) to remove cellular debris. Infective virus titre was determined by plaque assay and virus aliquots were stored at $-70^{\circ} \mathrm{C}$ until used.

\section{Plaque reduction neutralization assay}

The PRN assay was performed as has been described by Ho \& Babuik (1979) with some minor modifications. Briefly, serum specimens were heatinactivated at $56{ }^{\circ} \mathrm{C}$ for $30 \mathrm{~min}$ and serially diluted starting at 1:4 in Eagle's minimum essential medium (EMEM) in 96-well plates (12 $\mu \ell$ per well).

A standard inoculum of CDV was added to each well [12 $\mu \ell$ containing $50-70$ plaque-forming units (PFU)] and incubated at $37^{\circ} \mathrm{C}$ in air with $5 \% \mathrm{CO}_{2}$ for 180 min. The virus-serum mixtures were then added to duplicate Vero cell monolayers when approximately $90 \%$ confluent in 24 -well plates $(100 \mu \ell$ per well) and incubated at $37^{\circ} \mathrm{C}$ in $5 \% \mathrm{CO}_{2}$ for $60 \mathrm{~min}$. The inocula were then removed and the monolayers were covered with an overlay of $2 \%$ carboxymethyl cellulose in 2x EMEM ( $1 \mathrm{~m} \ell$ per well). The plates were incubated for 4 days at $37^{\circ} \mathrm{C}$ in air with $5 \%$ $\mathrm{CO}_{2}$. The monolayers were stained with neutral red and plaques counted visually on day 5 . The PRN values were determined using the Karber formula (Ballew 1986) and the serum titre required to reduce the number of PFU by $90 \%$ was determined.

\section{RESULTS}

Twenty-three of the 50 dogs that were tested had verifiable vaccination records while 27 of them did not. Of the 23 vaccinated dogs, only four $(17.4 \%)$ had PRN antibody titres of $<1: 100$, three $(13.0 \%)$ had titres less than $1: 1000$, and $16(69.6 \%)$ had PRN titres $>1: 1000$. Of the 27 dogs with no vaccination records, nine (33.3\%) had PRN titres $<1: 100$, four $(14.8 \%)$ had titres $<1: 1000$, while 14 $(51.9 \%)$ had titres $>1: 1000$.

A one-tailed student $t$ test done on the mean PRN titre of the two groups gave a $P$ value $\leq 0.020882$. This shows a significant difference in the PRN values of the two groups. Considering a titre of $<1: 100$ as the arbitrary cut-off for protection, vaccinated dogs had a much higher protective titre than the 
TABLE 1 History and PRN antibody titres of vaccinated dogs

\begin{tabular}{|c|c|c|c|c|c|}
\hline S/no. & Sample no. & Age (in months) & Sex & Breed & PRN titre \\
\hline 1 & N188 & 3 & $\mathrm{M}$ & Alsation* & 1573.9 \\
\hline 2 & N182 & 4 & $\mathrm{~F}$ & Alsation & 3.0 \\
\hline 3 & N205 & 4 & M & Alsation & 73.3 \\
\hline 4 & N247 & 4 & $\mathrm{M}$ & Alsation & 0.0 \\
\hline 5 & N185 & 5 & $\mathrm{~F}$ & Alsation & 5066.0 \\
\hline 6 & N230 & 5 & $\mathrm{M}$ & Alsation & 2339.0 \\
\hline 7 & CD009 & 6 & $\mathrm{M}$ & Cross & 27.3 \\
\hline 8 & CD007 & 9 & $\mathrm{~F}$ & Alsation & 5559.7 \\
\hline 9 & CD008 & 9 & $\mathrm{M}$ & Alsation & 4377.3 \\
\hline 10 & N238 & 10 & $\mathrm{~F}$ & Alsation & 5559.7 \\
\hline 11 & CD002 & 11 & $\mathrm{~F}$ & Alsation + Labrador & 5066.0 \\
\hline 12 & $1016 / 573$ & 12 & $\mathrm{M}$ & Alsation & 2344.5 \\
\hline 13 & N263 & 12 & $\mathrm{~F}$ & Alsation & 910.8 \\
\hline 14 & CD010 & 12 & $\mathrm{M}$ & Alsation & 1723.2 \\
\hline 15 & 527 & 15 & $\mathrm{M}$ & Alsation & 4740.5 \\
\hline 16 & 559 & 15 & $\mathrm{~F}$ & Alsation & 4150.8 \\
\hline 17 & CD012 & 15 & $\mathrm{~F}$ & Alsation & 774.7 \\
\hline 18 & OLD313 & 21 & $\mathrm{~F}$ & Alsation & 5202.4 \\
\hline 19 & L/024 & 30 & $\mathrm{~F}$ & Alsation & 1637.9 \\
\hline 20 & 704 & 32 & $\mathrm{~F}$ & Alsation & 5066.0 \\
\hline 21 & OLD051 & 34 & $\mathrm{~F}$ & Terrier & 1374.8 \\
\hline 22 & CD004 & 36 & $\mathrm{~F}$ & Alsation & 724.9 \\
\hline 23 & CD011 & 36 & $\mathrm{M}$ & Alsation & 3492.4 \\
\hline
\end{tabular}

* Alsation = German shepherd

unvaccinated groups. Of the 27 unvaccinated dogs, $14(51.9 \%)$ had PRN titres higher than 1:1000. This is a rather high figure in unvaccinated dogs and is more than likely an indication of previous exposure to a wild type strain.

There is a decline in the PRN titres of dogs less than 5 months of age and from 9 months, when they are usually given a second dose of vaccine, there is an increase in PRN titre. This increase was sustained throughout the age groups from 9 months to 3 years.

\section{DISCUSSION}

Serological testing for the prevalence of CDV antibodies has not been done on a routine basis in Nigeria but the CDV (combined) vaccine has been used for several years. We have shown in this study that $37(54 \%)$ of 50 dogs tested had antibodies to CDV, using an arbitrary PRN titre $>100$ as a cut-off for sero-positivity. In addition, using sero-positivity as a measure of protection, we showed that of 23 vaccinated dogs, four had specific CDV PRN titres less than the minimum protective level.

The report of Von Messling, Harder, Moenning, Rautenberg, Nolte \& Hass (1999) suggested that a virus-neutralizing titre of 1:100 correlated with protection against CDV infection. On the basis of this suggestion, only four of 23 vaccinated dogs had specific PRN titres less than the minimum protective level, indicating that they were not protected following vaccination. Suboptimal titres or loss of infectivity of vaccine virus owing to electrical power failures or faults in handling, transportation and storage of vaccines are probable reasons for this observation. It is noteworthy that these four dogs fall within the 3-6 months age range. In the second group of dogs with no vaccination record, ten out of 27 had PRN titres $<1: 100$. This shows a significant difference in the vaccinated group as compared to the unvaccinated group. High PRN titres of $>1000$ were recorded in 14 of the 27 unvaccinated dogs. These high antibody titres in unvaccinated dogs are an indication of previous exposure to canine distemper virus and this would most likely be a wild type strain. However, these results are being interpreted with caution since it may be very difficult to reasonably correlate titres obtained using the PRN test and virus neutralization with protection from infection following exposure (Twark \& Dodds 2000; Palmquist 2000). Nonetheless, a widespread use of the PRN test in serological surveys of CDV infection will provide more reproducible data that can be used to generate a bank of internationally standardized CDV-neutralizing sera in future. Follow-up prospective investigation of CDV infection in the dogs examined in the present report will go a long 
way to help establish the minimum protective PRN test titres that may be important in such international effort. Further serological studies are also necessary to fully understand the epizootiology of CDV in Nigeria and better evaluate the effectiveness of current vaccination schedules.

\section{REFERENCES}

BALLEW, H.C. 1986. Neutralization, in Clinical virology manual, edited by S. Specter \& G.J. Lancz. New York: Elsevier Science.

FENNER, F.J., GIBBS, E.P.J., MURPHY, F.A., ROTT, R., STUDDERT, M.J., WHITE, D.O. 1993. Veterinary Virology. $2^{\text {nd }}$ ed. London: Academic Press.

GREENE, C.E. 1990. Immunoprophylaxis and immunotherapy, in Infectious diseases of the dog and cat, edited by C.E. Greene. Philadelphia: W.B. Saunders.

HO, C.K. \& BABIUK, L.A. 1979. A new plaque system for canine distemper: characteristics of the green strain of canine distemper virus. Canadian Journal of Microbiology, 25:680-685.

HORST, J.C. 1975. Diseases of dogs. New York: Pergamon Press.

IWATSUKI, K., MIYASHITA, N., YOSHIDA, E., GEMMA, T., SHIM, Y.S. \& MORI, T. 1999. Molecular and phylogenetic analyses of the haemagglutinin $(\mathrm{H})$ proteins of field isolates of canine distemper virus from naturally infected dogs. Journal of General Virology, 78:373-380.

LEE, M.S., COHEN, B., HAND, J. \& NOKES, D.J. 1999. A simplified and standardized neutralization enzyme immunoassay for the quantification of measles neutralizing antibody. Journal of Virological Methods, 78:209-217.

McCAW, D.L., THOMPSON, M., TATE, D., BONDERER, A. \& CHEN, Y. 1998. Serum distemper virus and parvovirus anti- body titres among dogs brought to a veterinary hospital for revaccination. Journal of the American Veterinary Medical Association, 213:72-75.

PALMQUIST, R. 2000. Opposing views of revaccination strategies. Journal of the American Veterinary Medical Association, 217:1789-1791.

SHOPE, R.E. 1990. Antigen and antibody detection and update on the diagnosis of dengue. Southeast Asian Journal of Tropical Medicine \& Public Health, 21:642-645.

SMITH, C.A. 1995. Current concepts: are we vaccinating too much? Journal of the American Veterinary Medical Association, 207: 421-425.

SOMA, T., ISHII, H., HARA, M., YAMAMOTO, S., YOSHIDA, T., KINOSHITA, T. \& NOMURA, K. 2001. Comparison of immunoperoxidase plaque staining and neutralizing tests for canine distemper virus. Veterinary Research Communications, 25:311-325.

TWARK, L. \& DODDS, W.J. 2000. Clinical use of serum parvovirus and distemper virus antibody titers for determining revaccination strategies in healthy dogs. Journal of the American Veterinary Medical Association, 217:1021-1024.

VON MESSLING, V., HARDER, T.C., MOENNING, V., RAUTENBERG, P., NOLTE, I. \& HASS, L. 1999. Rapid and sensitive detection of immunoglobulin M (IgM) and IgG antibodies against canine distemper virus by a new recombinant nucleocapsid protein-based enzyme-linked immunosorbent assay. Journal of Clinical Microbiology, 37:1049-1056.

WAKASA, C., IWATSUKI, K., OHASHI, K., NAKAMURA, K. \& $\mathrm{KAI}, \mathrm{C} .2000$. Sequence analysis of the genes encoding the phosphoprotein of recent isolates of canine distemper virus in Japan. Journal of Veterinary Medical Sciences, 62:97101.

YOSHIDA, E., SHIN, Y.S., IWATSUKI, K., GEMMA, T., MIYASHITA, N., TOMONAGA, K., HIRAYAMA, N., MIKAMI, T. \& KAI, C. 1999. Epitopes and nuclear localization analysis of canine distemper virus nucleocapsid protein by expression of its deletion mutants. Veterinary Microbiology, 66:313-320. 